\title{
Prevalence and key radiographic spinal malalignment parameters that influence the risk for gastroesophageal reflux disease in patients treated surgically for adult spinal deformity
}

\author{
Tetsuro Ohba*, Shigeto Ebata, Kensuke Koyama and Hirotaka Haro
}

\begin{abstract}
Background: Gastroesophageal reflux disease (GERD) is a factor that has a significant negative impact on the quality of life (QoL). Vertebral fractures and/or spinal malalignment may influence the frequency of GERD. However, the epidemiology and pathology of GERD in patients with adult spinal deformity (ASD) are still largely unknown. To establish the optimal surgical strategy for GERD in patients treated surgically for ASD, we sought to clarify the GERD prevalence, determine radiographically which spinal malalignment parameters influence GERD risk, and evaluate GERD improvement postoperatively.
\end{abstract}

Methods: Seventy-one consecutive patients with ASD who were treated with thoracolumbar corrective surgery and followed up for at least 1 year were enrolled. GERD was diagnosed by a gastroenterologist based on proton pump inhibitor medication response and/or an FSSG score $>8$ points. Full-length lateral radiographs in a standing posture and in a supine, fulcrum backward-bending (FBB) position were taken preoperatively and 1 year postoperatively, and radiographic parameters were obtained. Correlations between radiographic parameters and FSSG scores were determined by Pearson's correlation coefficient. Multivariate logistic regression analyses were performed to evaluate the odds ratio $(\mathrm{OR})$ with a $95 \%$ confidence interval $(95 \% \mathrm{Cl})$ for potential risk factors for GERD.

Results: Patients were classified into two groups based on GERD symptoms, with 37 (52\%) in the GERD+ group. Thoracolumbar kyphosis (TLK) in the FBB position was significantly more common in the GERD+ versus the GERDgroup. Multivariate logistic regression analysis showed that lumbar lordosis (LL) and TLK curve in the FBB position significantly influenced the presence of GERD. Other factors showed no association with GERD. Significant improvements in FSSG scores were noted 1 year postoperatively. However, 20 (28.2\%) patients still had GERD symptoms. The postoperative TLK curve was highly significantly correlated with FSSG scores 1 year postsurgery.

Conclusions: Of the 71 patients treated surgically for ASD, 37 (52\%) had a high frequency of GERD symptoms. An inflexible thoracolumbar curve with increased TLK in the FBB position was significantly associated with GERD symptoms. Despite significant improvements in FSSG scores postoperatively, insufficient correction of TLK might be a risk factor for persistent GERD symptoms.

Keywords: Adult spinal deformity, Gastroesophageal reflux disease, Thoracolumbar kyphosis, Fulcrum backwardbending position, Surgical spinal correction, Surgical planning

\footnotetext{
* Correspondence: tooba@yamanashi.ac.jp

Department of Orthopaedics, University of Yamanashi, 1110, Shimokato,

Chuo, Yamanashi 409-3898, Japan
} 


\section{Background}

In an aging society, adult spinal deformity (ASD) remains one of the most controversial topics within the field of spinal disorders due to the significant impact it can have on health-related quality of life (HRQoL). Spinal malalignment in the sagittal plane has been correlated with pain as well as physical and mental disability $[1,2][3,4]$. Therefore, numerous studies have attempted to establish mathematical prediction formulas for optimal surgical planning in the treatment of ASD. Attention has been paid to the correlation between radiographic spinopelvic parameters and HRQoL scores evaluated by questionnaires such as the Oswestry Disability Index (ODI), the Scoliosis Research Society-22r, and the Short Form [SF]-12 [4-7].

Gastroesophageal reflux disease (GERD) is recognized as a factor that has a significant negative impact on quality of life (QoL) [8], and a recent large survey showed that $37.6 \%$ of Japanese individuals were affected by GERD [9]. In addition, it has been suggested that vertebral fractures and/or spinal malalignment may influence the frequency of GERD [10-13]. However, there are few studies about the epidemiology and pathology of GERD in patients with severe spinal deformity who require treatment with thoracolumbar corrective surgery. Additionally, the efficacy of surgical spinal correction for GERD is still largely unknown. To establish the optimal surgical strategy for treating GERD in patients with ASD, we set out to do the following: 1) to clarify the prevalence of GERD; 2) to determine radiographically which spinal malalignment parameters predominantly influence the risk for GERD; and 3) to evaluate the improvement of GERD after surgical spinal correction.

\section{Methods}

\section{Patients and surgical techniques}

All patients were considered candidates for thoracolumbar correction if a fusion was indicated because of ASD and if a full course of conservative care had been exhausted. The inclusion criteria were age greater than 60 years and a radiographic diagnosis of ASD defined by at least one of the following parameters: a coronal Cobb angle more than $30^{\circ}$; a C7 sagittal vertical axis (SVA), which is the distance between the $\mathrm{C} 7$ plumb line and the posterosuperior edge of $\mathrm{S} 1$, more than $5 \mathrm{~cm}$; pelvic tilt (PT), which is the orientation of the pelvis with respect to the femurs and the rest of the body, more than $25^{\circ}$; and/or thoracic kyphosis (TK) more than $30^{\circ}$. Patients were excluded if they had inflammatory arthritis, tumors, or neuromuscular diseases. The following information was obtained from each subject: number of vertebral fractures; radiographic parameters (as described below); intake of nonsteroidal anti-inflammatory drugs (NSAIDs), and intake of bisphosphonates. The demographic details of the patients are shown in Table 1.

Seventy-three patients were treated with thoracolumbar corrective surgery between April 2010 and March 2014 by three board-certified spinal surgeons at a single institution; two patients missed follow-up. Therefore, 71 consecutive patients with ASD who were treated with thoracolumbar corrective surgery and followed up for a minimum of 1 year were enrolled in this study.

\section{Radiographic measurements}

Radiographic data collection consisted of full-length lateral radiographs obtained with the patient in a freestanding posture with fingers placed on the clavicles or supine in a fulcrum backward-bending (FBB) position preoperatively. Preoperative evaluation for flexibility of the thoracolumbar spine is essential to perform the optimum surgery and includes an assessment of the necessity of osteotomy and/or range of fusion. Radiographs obtained with the supine patient in an FBB position is an effective technique to evaluate the flexibility of the thoracolumbar kyphotic curvature [14].

Recent reports indicated that the following sagittal spinopelvic parameters are directly correlated with HRQoL scores evaluated by questionnaires such as the ODI, the Scoliosis Research Society-22r, and the SF-12 [4-7]: the SVA, PT, and pelvic incidence-lumbar lordosis (PI-LL) have been known as the parameters that are most strongly correlated with disability of patients with ASD [3]. Standing full-length lateral radiographs were captured 1 year postoperatively. The following radiographic parameters were measured using a lateral view: T5-T12 TK, T10-L2 thoracolumbar kyphosis (TLK), T12-S1 LL angles, PI, PT, sacral slope (SS), and SVA. Kyphosis was expressed as a positive value, and lordosis was expressed as a negative value. Using a frontal view radiograph with the patient in the standing position, we measured the coronal Cobb angle in the thoracolumbar, lumbar and thoracic spine. Vertebral fracture was considered present if at least one of three height measurements (anterior, middle, and posterior) for one vertebra had decreased by $>20 \%$ compared with the height of the nearest uncompressed vertebral body [15]. Radiographic measurements were obtained by one of the authors based on a single measurement using standardized techniques. This author has more than 10 years of experience in spinal surgery and was blinded to the Quest scores.

\section{Diagnosis of GERD}

All patients were asked to respond to the Frequency Scale for Symptoms of GERD (FSSG) questionnaire regardless of any specific complaints. The questionnaire contains 12 questions and has been recently developed as a self-report instrument that is written in simple and 
Table 1 Baseline characteristics of patients with ASD with and without GERD

\begin{tabular}{|c|c|c|c|c|}
\hline Variable & Overall $(n=71)$ & $\mathrm{GERD}+(n=37)$ & GERD- $(n=34)$ & $P$ value \\
\hline Age (y) & $70.4 \pm 7.4$ & $70.8 \pm 6.54$ & $70.0 \pm 8.23$ & 0.6663 \\
\hline Female/male gender (n) & $62 / 9$ & $32 / 5$ & $30 / 4$ & 0.7297 \\
\hline TK, standing $\left(^{\circ}\right)$ & $24.9 \pm 19.0$ & $29.3 \pm 19.8$ & $20.7 \pm 17.3$ & 0.0620 \\
\hline TLK, standing $\left(^{\circ}\right)$ & $19.34 \pm 18.4$ & $23.7 \pm 19.7$ & $15.2 \pm 16.1$ & 0.0561 \\
\hline $\mathrm{LL}$, standing $\left(^{\circ}\right)$ & $13.2 \pm 19.6$ & $14.4 \pm 18.2$ & $12.2 \pm 21.7$ & 0.6571 \\
\hline $\mathrm{PI}\left({ }^{\circ}\right)$ & $53.1 \pm 9.6$ & $54.4 \pm 11.2$ & $51.9 \pm 8.1$ & 0.2963 \\
\hline $\mathrm{PT}\left({ }^{\circ}\right)$ & $37.5 \pm 10.6$ & $38.4 \pm 10.0$ & $36.5 \pm 11.2$ & 0.4759 \\
\hline SS $\left(^{\circ}\right)$ & $17.0 \pm 11.9$ & $16.9 \pm 10.1$ & $17.2 \pm 13.4$ & 0.8947 \\
\hline SVA (mm) & $104.8 \pm 64.6$ & $116.9 \pm 56.8$ & $93.1 \pm 71.0$ & 0.1354 \\
\hline $\operatorname{PI}-\mathrm{LL}\left({ }^{\circ}\right)$ & $29.3 \pm 16.5$ & $27.52 \pm 17.3$ & $31.0 \pm 15.4$ & 0.3864 \\
\hline Cobb angle $\left(^{\circ}\right)$ & $23.6 \pm 16.9$ & $26.9 \pm 17.9$ & $20.1 \pm 15.1$ & 0.0918 \\
\hline $\mathrm{TK}, \mathrm{FBB}\left(^{\circ}\right)$ & $19.9 \pm 15.8$ & $24.2 \pm 16.4$ & $14.8 \pm 13.6$ & $<0.05$ \\
\hline TLK, FBB $\left(^{\circ}\right)$ & $7.2 \pm 14.4$ & $13.6 \pm 15.2$ & $-0.42 \pm 8.8$ & $<0.0001$ \\
\hline $\mathrm{LL}, \mathrm{FBB}\left(^{\circ}\right)$ & $31.9 \pm 16.3$ & $29.9 \pm 15.8$ & $34.3 \pm 17.0$ & 0.0918 \\
\hline Cobb angle $\sim 30^{\circ} / 30^{\circ} \sim(\mathrm{n})$ & & $22 / 15$ & $29 / 5$ & $<0.05$ \\
\hline Number of thoracolumbar vertebral fractures ( $n$ ) & $0.55 \pm 1.1$ & $0.78 \pm 1.3$ & $0.32 \pm 0.84$ & 0.0876 \\
\hline Intake of NSAIDs & $15(21.1 \%)$ & $10(27.02 \%)$ & $5(14.7 \%)$ & 0.252 \\
\hline Intake of bisphosphonates & $8(11.3 \%)$ & $6(16.2 \%)$ & $2(5.88 \%)$ & 0.264 \\
\hline Intake of PPI & $34(47.9 \%)$ & $34(91.9 \%)$ & 0 & \\
\hline
\end{tabular}

Data are mean \pm standard deviation unless otherwise shown Bolded values with $P<0.05$

$A S D$ adult spinal deformity, FBB fulcrum backward-bending, GERD gastroesophageal reflux disease, $L L$ lumbar lordosis, $T K$ thoracic kyphosis, $T L K$ thoracolumbar kyphosis, PI pelvic incidence; PT pelvic tilt, SS sacral slope, SVA sagittal vertical axis, NSAIDs non-steroidal anti-inflammatory drugs, PPI Proton pump inhibitor

easy-to-understand language. Symptom frequency was measured on the following scale: never $=0$; occasionally $=$ 1 ; sometimes $=2$; often $=3$; and always $=4$. The FSSG questionnaire is appropriate for management of GERD in general practice. The results from the FSSG questionnaire have been shown to correlate strongly with endoscopic findings; thus, endoscopy is not a requirement [16].

Evaluation of GERD was conducted within 2 weeks prior to surgery and 1 year after surgical spinal correction. GERD was diagnosed by a gastroenterologist based on the patient's response to proton pump inhibitor (PPI) medication and/or an FSSG score $>8$ points $[13,16]$, and patients were divided into groups based on the presence or absence of GERD, that is, GERD+ and GERD-, respectively.

\section{Statistical analyses}

All data are reported as means \pm SD. Data were analyzed using a two-sided Student $t$-test and a Fisher exact test to determine significant differences. Correlations between radiographic parameters and FSSG scores were determined by Pearson's correlation coefficient. All statistical calculations were performed with Prism version 6.0 (Graph Pad Software, La Jolla, CA). Multivariate logistic regression analyses were performed with $\mathrm{R}$ software, version 3.2 .3 , to evaluate the odds ratio (OR) with a 95\% confidence interval (95\% CI) for potential risk factors for GERD. For all tests,
$P<0.05$ was considered statistically significant (" $P<0.05$, $\left.{ }^{* *} P<0.005,{ }^{* * *} P<0.0005,{ }^{* * * * *} P<0.0001\right)$.

\section{Results}

Preoperative prevalence of GERD and patient characteristics

The mean age of the patients in the study population was $70.6 \pm 7.3$ years, and there were 62 females and 9 males. Of the 71 patients treated surgically for ASD, 37 (52\%) had a high frequency of GERD symptoms. Table 1 summarizes the preoperative baseline characteristics and spinal radiographic parameters measured in the patients in the GERD+ and GERD- groups. There were no significant between-group differences in mean age, gender balance, intake of oral NSAIDs, oral bisphosphonates or number of thoracolumbar vertebral fractures (Table 1). Of the 37 patients in the GERD+ group, 34 (91.9\%) were treated with a PPI prescribed by a gastroenterologist (Table 1). In accordance with past reports [17], our data also showed that patients with a coronal Cobb angle of more than $30^{\circ}$ had GERD symptoms more frequently (Table 1). There were no significant differences between the groups for any of the sagittal spine radiographic parameters measured in a standing position. In contrast, TK and TLK in the FBB position were significantly 
larger in the GERD+ group compared with the respective values in the GERD- group (Table 1).

Strong correlation coefficients were observed between TK, TLK and LL in the FBB position and in the standing position. Therefore, the multivariate logistic regression analysis was conducted twice, once with all variables excluding TK, TLK and LL in the FBB position (Table 2) and once excluding TK, TLK, and LL in the standing position (Table 3). Parameters used in the multivariate analysis were age, sex, SVA, TK, TLK, LL, PI, PT, SS and Cobb angle. The smaller LL and larger TLK curves in the FBB position and the larger coronal Cobb angle in the standing position were significantly associated with the presence of GERD (Table 3). No other variables, including sagittal parameters, were significantly correlated. Representative cases with or without severe GERD are shown in Fig. 1. Both cases had definite sagittal imbalance, including large TLK in the standing position. In contrast, only the GERD+ case had a large fixed TLK curve in the FBB position (Fig. 1b).

\section{Efficacy of surgical spinal correction for GERD}

One year after surgery, the total FSSG score of ASD patients decreased significantly from $14.5 \pm 11.8$ (presurgery) to $6.4 \pm 5.5$ (postsurgery) $(P<0.0001)$ (Fig. 2a). Additionally, both the acid-related score and the dysmotility portion of the FSSG score decreased significantly $(P<0.0001)$ (Fig. 2b, c). However, of the 71 patients treated surgically for ASD, 20 (28.2\%) still had GERD symptoms (FSSG score $>8$ points) 1 year after surgery.

Correlation between FSSG scores and TLK curves.

The correlations between FSSG scores and TLK angles are shown in Fig. 3. No significant correlation was found between preoperative FSSG scores and preoperative standing TLK curves (Fig. 3a). In contrast, a significant positive correlation was found between preoperative FSSG scores and preoperative TLK curves in the FBB

Table 2 Multivariate logistic regression analysis of risk factors for developing GERD symptoms based on radiographs in the standing position

\begin{tabular}{llll}
\hline Parameter & OR & $95 \% \mathrm{Cl}$ & $P$-value \\
\hline TK, standing & 1.028 & $0.972-1.093$ & 0.2266 \\
TLK, standing & 1.020 & $0.967-1.080$ & 0.3443 \\
LL, standing & 0.998 & $0.939-1.063$ & 0.9500 \\
PI -LL & 1.039 & $0.9477-1.167$ & 0.4332 \\
PT & 0.982 & $0.882-1.067$ & 0.6832 \\
SS & 0.939 & $0.832-1.034$ & 0.2225 \\
SVA & 0.999 & $0.984-1.013$ & 0.8371 \\
Cobb angle & 1.099 & $0.997-1.239$ & 0.0727 \\
\hline
\end{tabular}

Cl confidence interval, GERD gastroesophageal reflux disease, $L L$ lumbar lordosis, OR odds ratio, TK thoracic kyphosis, TLK thoracolumbar kyphosis, PI pelvic incidence; PT pelvic tilt, SS sacral slope, SVA sagittal vertical axis
Table 3 Multivariate logistic regression analysis of risk factors for developing GERD symptoms based on radiographs in the FBB position

\begin{tabular}{llll}
\hline Parameter & OR & $95 \% \mathrm{Cl}$ & $P$ value \\
\hline TK, FBB & 1.063 & $0.982-1.171$ & 0.1546 \\
TLK, FBB & 1.192 & $1.065-1.397$ & $<0.01$ \\
LL, FBB & 0.877 & $0.776-0.962$ & $<0.05$ \\
PI-LL & 1.018 & $0.901-1.209$ & 0.7701 \\
PT & 1.035 & $0.872-1.164$ & 0.5870 \\
SS & 1.017 & $0.859-1.171$ & 0.8111 \\
SVA & 0.996 & $0.978-1.015$ & 0.6842 \\
Cobb angle & 1.217 & $1.051-1.495$ & $<0.05$
\end{tabular}

Bolded values with $P<0.05$

$\mathrm{Cl}$ confidence interval, FBB fulcrum backward-bending, GERD gastroesophageal reflux disease, $L L$ lumbar lordosis, $O R$ odds ratio, $T K$ thoracic kyphosis, $T L K$ thoracolumbar kyphosis, PI pelvic incidence, PI pelvic tilt, SS sacral slope, SVA sagittal vertical axis

position (Fig. 3b). Surprisingly, a significant, highly positive correlation was found between FSSG scores and standing TLK 1 year after surgery $(r=0.66, P<0.0001)$ (Fig. 3c).

\section{Discussion}

Our data showed that of the 71 patients who underwent surgical treatment for ASD, 37 (52\%) had a high frequency of GERD symptoms. Therefore, GERD might be one of the main complaints to evaluate when performing thoracolumbar corrective surgery. Spinal malalignment parameters may influence the risk for GERD in patients with ASD; therefore, precise information regarding these parameters should be obtained.

Age, female sex, obesity, hiatal hernia, smoking, regular consumption of alcohol, and $H$. pylori negativity have been elucidated as risk factors for GERD [8] [18-20]. In addition, the association between osteoporosis/spinal hyperkyphosis and increased prevalence of GERD has been recognized [10, 11, 13, 21]. However, these reports lacked detailed information on global spinal alignment, the location of the kyphosis, and the kyphosis angle. In accordance with past reports [17], our data also showed that scoliosis was a significant risk factor for GERD, and risk increased with a curve $>30$ degrees. A recent report on volunteers who attended a basic health checkup and had an evaluation of global spinal alignment indicated that the angle of the LL, sagittal balance, and back muscle strength are significant factors related to the presence of GERD [22]. In contrast to previous studies, we did not find spinal sagittal alignment based on parameters of TK, TLK, or LL measured by full-length standing spinal radiographs to be significant risk factors for GERD symptoms. This may be because our subjects were limited to patients who had serious spinal deformity. In the current study, PI-LL mismatch in the standing 

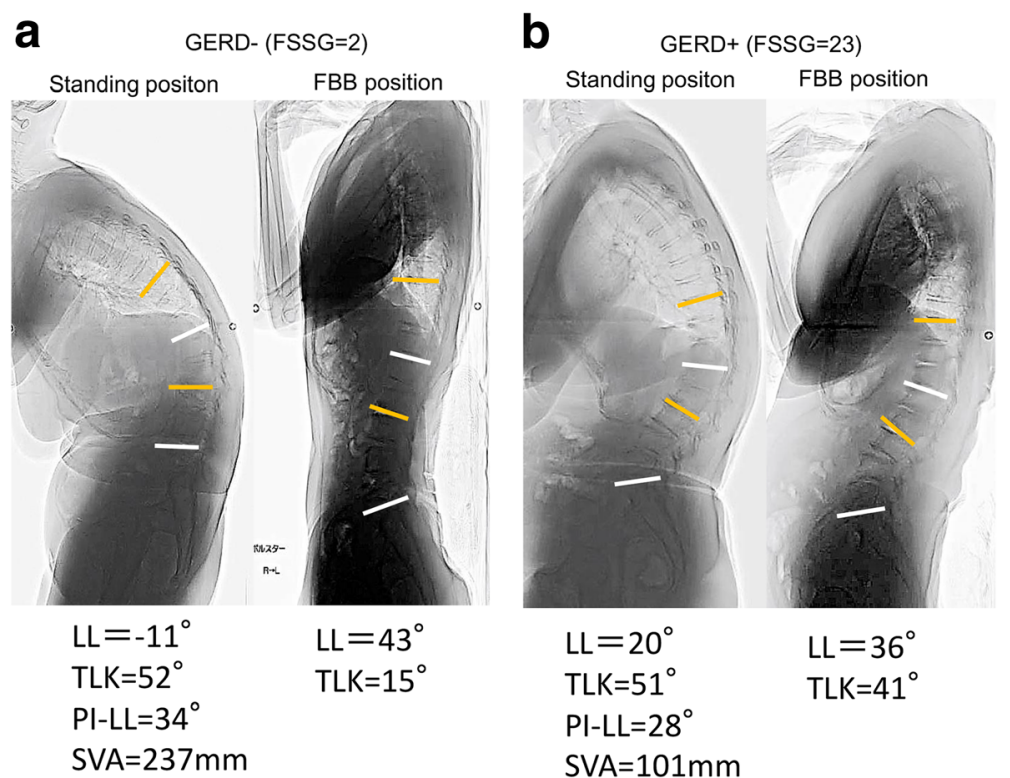

Fig. 1 Representative cases with or without severe gastroesophageal reflux disease symptoms: Frequency Scale for Symptoms of GERD score of 2 (a) or 23 (b). The white line denotes lumbar lordosis. The yellow line denotes thoracic lumbar kyphosisa Lateral standing radiograph showing global sagittal malalignment due to $\mathrm{a}-11^{\circ}$ lumbar lordosis and a $51^{\circ}$ thoracic lumbar kyphosis. Lateral radiograph obtained in the fulcrum backward-bending position showing thoracic lumbar kyphosis reduced to $15^{\circ}$. b Lateral standing radiograph showing a $-20^{\circ}$ lumbar lordosis and global sagittal malalignment due to a $51^{\circ}$ thoracic lumbar kyphosis. Lateral radiograph obtained in the fulcrum backward-bending position showing a $41^{\circ}$ thoracic lumbar kyphosis that is not flexible.

position also did not correlate with GERD symptoms (Table 1). The pathology underlying ASD can include a wide variety of spinal curves, such as marked global sagittal malalignment due to lumbar kyphosis or flexible or rigid TK and severe scoliosis without marked global sagittal malalignment [14]. Preoperative evaluation of the flexibility of the curvature of the spine is essential for selecting the optimal surgical strategy for patients with ASD, and it has been demonstrated that radiographs in the $\mathrm{FBB}$ position can be used to assess vertebral curve flexibility [14, 23, 24]. Our study showed that decreased LL and increased TLK measured in the FBB position, which indicate decompensatory sagittal malalignment, are critical risk factors for the development of GERD symptoms in patients with ASD (Fig. 1a, b).

A recent report indicated improvement of GERD in patients with spinal kyphotic deformity who underwent surgical spinal correction. However, this study did not consider the relationship between the improvement of a

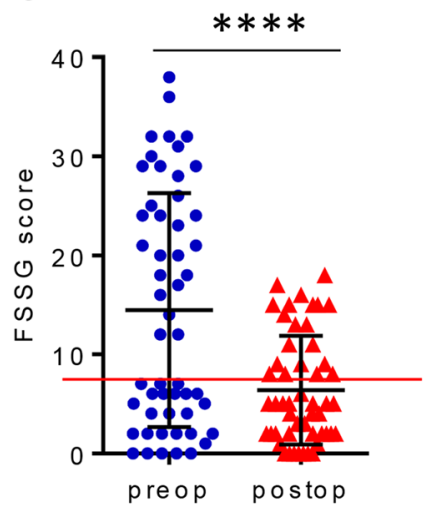

b Acid-related score

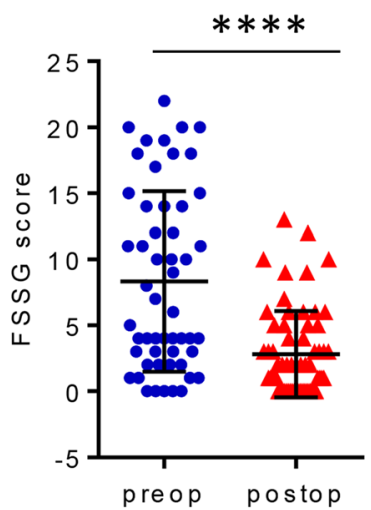

C Dysmotility score

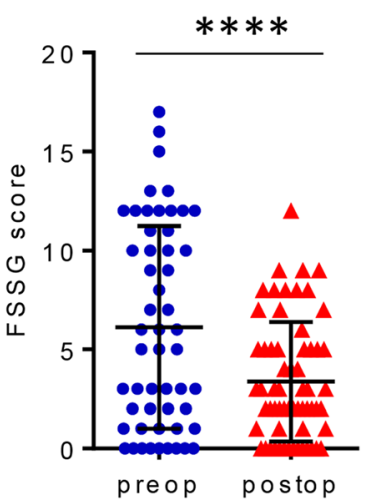

Fig. 2 a Comparison of preoperative and 1-year postoperative (preop and postop) FSSG scores $\left({ }^{* * *} P<0.0001\right)$. b Comparison of preoperative and 1-year postoperative (preop and postop) acid-related score in the FSSG scores (**** $<0.0001)$. c Comparison of preoperative and 1-year postoperative (preop and postop) dysmotility score in the FSSG scores (**** $P<0.0001)$ 

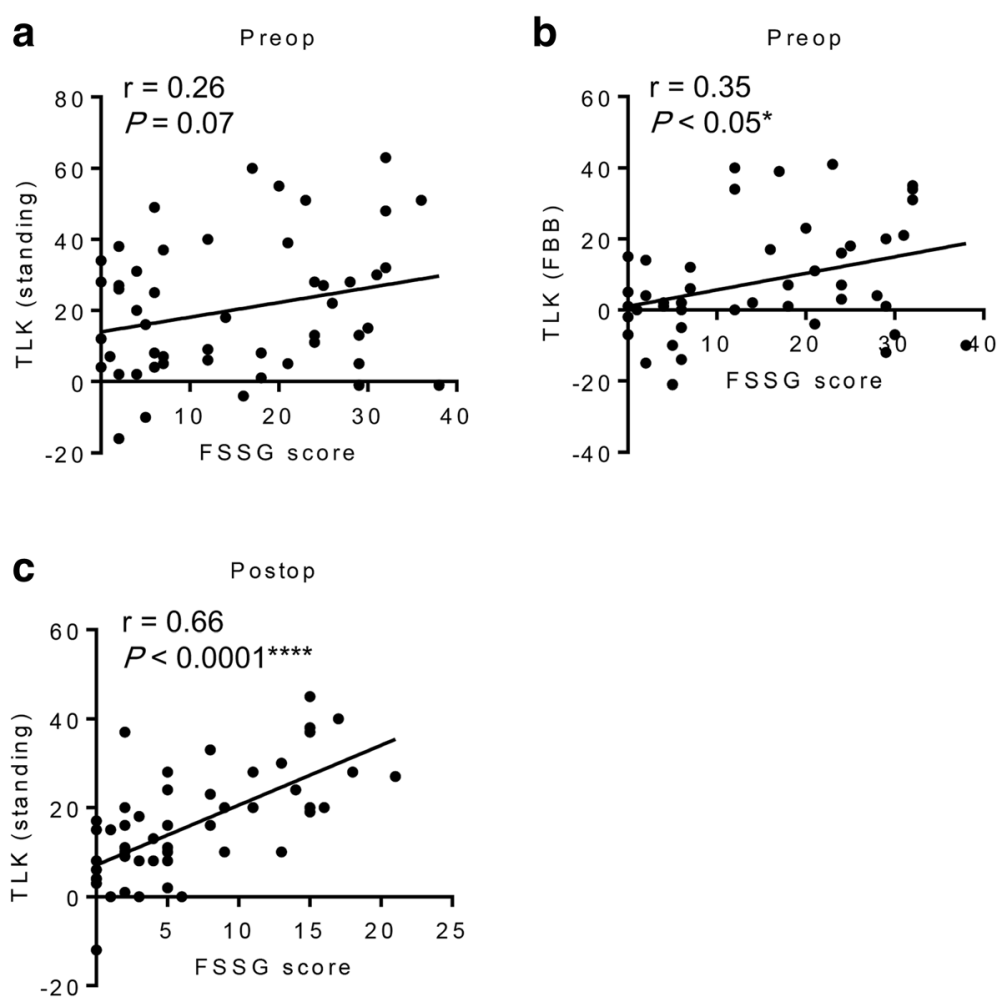

Fig. 3 a Correlation between preoperative FSSG scores and preoperative standing TLK curves. b Correlation between preoperative FSSG scores and preoperative TLK curves in the FBB position. c Correlation between the 1-year postoperative FSSG scores and the postoperative standing TLK curves

GERD symptoms and the global spinal alignment [25]. Similar to the recent report [25], our study also showed significant improvements in FSSG scores that were noted postoperatively. In addition, we found some patients still had GERD symptoms, and postoperative fixed TLK was significantly highly correlated with FSSG scores 1 year after surgery. One possible mechanism for these results is that an increase in intra-abdominal pressure is caused by thoracolumbar kyphosis, with subsequent compression of the esophagus and stomach cranially, and this mechanism is supported by past reports [11, 22].

The limitations of this study were the small sample size and the assessment of GERD symptoms by the Quest score only, without diagnostic endoscopy or other additional tests. The sensitivity of the FSSG for diagnosis of GERD is about 70\%. In addition, GERD included reflux esophagitis and non-erosive reflux esophagitis (NERD). In the current study, most patients grouped as GERD-positive were dosed with PPIs, and they may be patients with NERD or functional heartburn. Further study using endoscopic evaluation is needed to elucidate the epidemiology and pathology of GERD in ASD more precisely. Additionally, because some patients might stop the intake of PPIs postoperatively, the influence of postoperative intake of oral PPIs on FSSG score should be evaluated in a further study. However, to our knowledge, this is the first study to report the prevalence of GERD in patients with ASD, to demonstrate that a fixed thoracolumbar curve that is decreased in LL and increased in TLK in the FBB position is significantly associated with development of GERD symptoms in these patients, and to suggest that insufficient surgical correction of TLK might be a risk factor for persistent GERD symptoms. Despite it being well known that sagittal spinopelvic alignment is an important factor for optimal surgical planning, the results of the current study also indicated that management of an inflexible thoracolumbar curve is necessary to treat GERD symptoms in patients with ASD.

\section{Conclusions}

Of the 71 patients treated surgically for ASD, 37 (52\%) had a high frequency of GERD symptoms. An inflexible thoracolumbar curve with an increased TLK in the FBB position was significantly associated with GERD symptoms. Despite significant improvements in FSSG scores postoperatively, insufficient correction of TLK might be a risk factor for persistent GERD symptoms. 


\section{Abbreviations}

ASD: Adult spinal deformity; FBB: Fulcrum backward-bending;

GERD: Gastroesophageal reflux disease; HRQoL: Health-related quality of life; LL: Lumber Lordosis; NERD: Non-erosive reflux esophagitis;

NSAIDs: Nonsteroidal anti-inflammatory drugs; ODI: Oswestry disability index; PI: Pelvic incidence; PPI: Proton pump inhibitor; PT: Pelvic tilt; SS: Sacral slope; SVA: Sagittal vertical axis; TK: Thoracic kyphosis; TLK: Thoracolumbar kyphosis

\section{Acknowledgements}

None.

\section{Funding}

There are no funding sources that should be declared.

\section{Availability of data and materials}

The datasets used and/or analyzed during the current study are available from the corresponding author on reasonable request.

\section{Authors' contributions}

TO analyzed and interpreted the patient data and was a major contributor in writing the manuscript. SE developed the study design. KK collected the patients' data and performed the statistical analysis. $\mathrm{HH}$ was the editorial supervisor of this study. All authors read and approved the final manuscript.

\section{Ethics approval and consent to participate}

This study was approved by our institutional review board and the ethical committee of the University of Yamanashi School of Medicine, Chairperson Zentarou Yamagata (Application number 1183). We received informed consent from all eligible patients and the patient consent was written.

\section{Consent for publication}

Not applicable.

\section{Competing interests}

The authors declare that they have no competing interests.

\section{Publisher's Note}

Springer Nature remains neutral with regard to jurisdictional claims in published maps and institutional affiliations.

Received: 24 April 2017 Accepted: 4 January 2018

Published online: 10 January 2018

\section{References}

1. Lafage V, Schwab F, Patel A, Hawkinson N, Farcy JP. Pelvic tilt and truncal inclination: two key radiographic parameters in the setting of adults with spinal deformity. Spine (Phila Pa 1976). 2009:34(17):E599-606.

2. Rose PS, Bridwell KH, Lenke LG, Cronen GA, Mulconrey DS, Buchowski JM, Kim YJ. Role of pelvic incidence, thoracic kyphosis, and patient factors on sagittal plane correction following pedicle subtraction osteotomy. Spine (Phila Pa 1976). 2009;34(8):785-91.

3. Schwab F, Lafage V, Patel A, Farcy JP. Sagittal plane considerations and the pelvis in the adult patient. Spine (Phila Pa 1976). 2009;34(17):1828-33.

4. Yamato Y, Hasegawa T, Kobayashi S, Yasuda T, Togawa D, Arima H, Oe S, lida T, Matsumura A, Hosogane N, et al. Calculation of the target lumbar Lordosis angle for restoring an optimal pelvic tilt in elderly patients with adult spinal deformity. Spine (Phila Pa 1976). 2016;41(4):E211-7.

5. Glassman SD, Bridwell K, Dimar JR, Horton W, Berven S, Schwab F. The impact of positive sagittal balance in adult spinal deformity. Spine (Phila Pa 1976). 2005;30(18):2024-9.

6. Gum JL, Bridwell KH, Lenke LG, Bumpass DB, Sugrue PA, Karikari IO, Carreon LY. SRS22R appearance domain correlates most with patient satisfaction after adult deformity surgery to the sacrum at 5-year follow-up. Spine (Phila Pa 1976). 2015;40(16):1297-302

7. Schwab FJ, Blondel B, Bess S, Hostin R, Shaffrey Cl, Smith JS, Boachie-Adjei O, Burton DC, Akbarnia BA, Mundis GM, et al. Radiographical spinopelvic parameters and disability in the setting of adult spinal deformity: a prospective multicenter analysis. Spine (Phila Pa 1976). 2013;38(13):E803-12.

8. Nandurkar S, Talley NJ. Epidemiology and natural history of reflux disease. Baillieres Best Pract Res Clin Gastroenterol. 2000;14(5):743-57.
9. Watanabe T, Urita Y, Sugimoto M, Miki K. Gastroesophageal reflux disease symptoms are more common in general practice in Japan. World J Gastroenterol. 2007;13(31):4219-23.

10. Yamaguchi T, Sugimoto T, Yamada H, Kanzawa M, Yano S, Yamauchi M, Chihara K. The presence and severity of vertebral fractures is associated with the presence of esophageal hiatal hernia in postmenopausal women. Osteoporos Int. 2002;13(4):331-6.

11. Yamaguchi T, Sugimoto T, Yamauchi M, Matsumori $Y$, Tsutsumi M, Chihara K Multiple vertebral fractures are associated with refractory reflux esophagitis in postmenopausal women. J Bone Miner Metab. 2005;23(1):36-40.

12. Kusano M, Hashizume K, Ehara Y, Shimoyama Y, Kawamura O, Mori M. Size of hiatus hernia correlates with severity of kyphosis, not with obesity, in elderly Japanese women. J Clin Gastroenterol. 2008:42(4):345-50.

13. Miyakoshi N, Kasukawa Y, Sasaki H, Kamo K, Shimada Y. Impact of spinal kyphosis on gastroesophageal reflux disease symptoms in patients with osteoporosis. Osteoporos Int. 2009;20(7):1193-8.

14. Taneichi $\mathrm{H}$. Update on pathology and surgical treatment for adult spinal deformity. J Orthop Sci. 2016;21(2):116-23.

15. Orimo H, Shiraki M, Hayashi $Y$, Hoshino T, Onaya T, Miyazaki S, Kurosawa $H$, Nakamura T, Ogawa N. Effects of 1 alpha-hydroxyvitamin D3 on lumbar bone mineral density and vertebral fractures in patients with postmenopausal osteoporosis. Calcif Tissue Int. 1994:54(5):370-6.

16. Kusano M, Shimoyama Y, Sugimoto S, Kawamura O, Maeda M, Minashi K, Kuribayashi S, Higuchi T, Zai H, Ino K, et al. Development and evaluation of FSSG: frequency scale for the symptoms of GERD. J Gastroenterol. 2004; 39(9):888-91.

17. Hosogane N, Watanabe K, Yagi M, Kaneko S, Toyama Y, Matsumoto M. Scoliosis is a risk factor for Gastroesophageal reflux disease in adult spinal deformity. Clin Spine Surg. 2017;30(4):E480-4.

18. Furukawa N, Iwakiri R, Koyama T, Okamoto K, Yoshida T, Kashiwagi Y, Ohyama T, Noda T, Sakata H, Fujimoto K. Proportion of reflux esophagitis in 6010 Japanese adults: prospective evaluation by endoscopy. J Gastroenterol. 1999:34(4):441-4.

19. Hampel H, Abraham NS, El-Serag HB. Meta-analysis: obesity and the risk for gastroesophageal reflux disease and its complications. Ann Intern Med. 2005;143(3):199-211

20. Sakaguchi M, Oka H, Hashimoto T, Asakuma Y, Takao M, Gon G, Yamamoto M, Tsuji Y, Yamamoto N, Shimada M, et al. Obesity as a risk factor for GERD in Japan. J Gastroenterol. 2008:43(1):57-62.

21. Tsuchie H, Fukata C, Takahashi K, Miyakoshi N, Kobayashi A, Kasukawa Y, Shimada $Y$, Inoue $\mathrm{H}$. Impact of lumbar kyphosis on gastric myoelectrical activity and heart rate variability in a model using flexion posture in healthy young adults. Biomed Res. 2011;32(4):271-8.

22. Imagama S, Hasegawa Y, Wakao N, Hirano K, Hamajima N, Ishiguro N. Influence of lumbar kyphosis and back muscle strength on the symptoms of gastroesophageal reflux disease in middle-aged and elderly people. Eur Spine J. 2012;21(11):2149-57.

23. Li J, Hwang S, Wang F, Chen Z, Wu H, Li B, Wei X, Zhu X, Li M. An innovative fulcrum-bending radiographical technique to assess curve flexibility in patients with adolescent idiopathic scoliosis. Spine (Phila Pa 1976). 2013;38(24):E1527-32

24. Samartzis D, Leung Y, Shigematsu H, Natarajan D, Stokes O, Mak KC, Yao G, Luk KD, Cheung KM. Selection of fusion levels using the fulcrum bending radiograph for the Management of Adolescent Idiopathic Scoliosis Patients with alternate level pedicle screw strategy: clinical decision-making and outcomes. PLoS One. 2015;10(8):e0120302.

25. Sugimoto $M$, Hasegawa $T$, Nishino $M$, Sahara $S$, Uotani T, Ichikawa H, Kagami $T$, Sugimoto K, Yamato Y, Togawa D, et al. Improvement of gastroesophageal reflux disease in Japanese patients with spinal kyphotic deformity who underwent surgical spinal correction. Dig Endosc. 2016;28(1):50-8. 